\title{
Active Viscoelasticity of Odd Materials
}

\author{
Debarghya Banerjee $\oplus^{1,2}$ Vincenzo Vitelli, ${ }^{3,4,5}$ Frank Jülicher® ${ }^{2,6}$ and Piotr Surówka $\oplus^{2,7,8,{ }^{*}}$ \\ ${ }^{1}$ Max Planck Institute for Dynamics and Self-Organization, 37077 Göttingen, Germany \\ ${ }^{2}$ Max Planck Institute for the Physics of Complex Systems, 01187 Dresden, Germany \\ ${ }^{3}$ James Franck Institute, The University of Chicago, Chicago, Illinois 60637, USA \\ ${ }^{4}$ Department of Physics, The University of Chicago, Chicago, Illinois 60637, USA \\ ${ }^{5}$ Kadanoff Center for Theoretical Physics, The University of Chicago, Chicago, Illinois 60637, USA \\ ${ }^{6}$ Cluster of Excellence Physics of Life, TU Dresden, 01062 Dresden, Germany \\ ${ }^{7}$ Department of Theoretical Physics, Wroctaw University of Science and Technology, 50-370 Wrocław, Poland \\ ${ }^{8}$ Würzburg-Dresden Cluster of Excellence ct.qmat, Germany
}

(Received 18 March 2020; revised 17 February 2021; accepted 19 February 2021; published 29 March 2021)

\begin{abstract}
The mechanical response of active media ranging from biological gels to living tissues is governed by a subtle interplay between viscosity and elasticity. We generalize the canonical Kelvin-Voigt and Maxwell models to active viscoelastic media that break both parity and time-reversal symmetries. The resulting continuum theories exhibit viscous and elastic tensors that are both antisymmetric, or odd, under exchange of pairs of indices. We analyze how these parity violating viscoelastic coefficients determine the relaxation mechanisms and wave-propagation properties of odd materials.
\end{abstract}

DOI: 10.1103/PhysRevLett.126.138001

Materials at the macroscopic scales are often described either as a fluid or as a solid. Such idealized behaviors are insufficient to describe materials that exhibit more complex mesoscopic organization. This is, for example, the case for liquid crystals or gels, where the structure of matter is more intricate due to elongation or chirality of the constituents. In addition, dissipative or active processes may enter the description of microscopic building blocks. As a result, the macroscopic description of materials includes both fluid- and solidlike features - the interplay of these two elements is known as viscoelasticity (see, e.g., Ref. [1]). Viscoelasticity is a common phenomenon, described by rheology, that can be observed in polymer systems [2], metamaterials $[3,4]$, and various biological media $[5,6]$.

From the swimming strokes of sperm cells to intracellular flows, biological systems present a wide variety of cases where chiral symmetry is broken [7-12]. Additionally, biological systems are often driven away from thermodynamic equilibrium by chemical reactions that render the matter active at the molecular level [13-15]. Recent work on chiral active matter has shown that the presence of activity and chirality, breaking essential microscopic symmetries, leads to novel response functions and transport coefficients in active fluids and solids [16-29]. In the simplest incarnation,

Published by the American Physical Society under the terms of the Creative Commons Attribution 4.0 International license. Further distribution of this work must maintain attribution to the author(s) and the published article's title, journal citation, and DOI. Open access publication funded by the Max Planck Society. the two coefficients were dubbed odd viscosity [30-39] and odd elasticity [18]. The main goal of this Letter is to combine these two formulations through a systematic description of chiral active systems based on symmetry principles. This leads to a hydrodynamic theory of active odd viscoelastic solids and liquids, distinguished by a long-time response to static and dynamic deformations. Odd responses are absent in standard Kelvin-Voigt and Maxwell models.

Basic viscoelastic models.-Viscoelasticity emerges as a consequence of complex phenomena at different scales. It is usually not possible to have a first principle analysis, and various phenomenological simplifications are employed. Since viscoelasticity can be viewed as a transient phenomenon to a time-independent state, we need to make some assumptions about the long-time behavior of our viscoelastic system. It can be either fluid or solid. These two distinct limiting cases of viscoelastic behavior are commonly described by the Maxwell and Kelvin-Voigt models, respectively. These models can be presented by a spring and a viscous damper in series (for Maxwell materials) or in parallel (for Kelvin-Voigt materials). The Kelvin-Voigt model typically defines a viscoelastic solid and captures strain relaxation. Maxwell materials define viscoelastic fluids, representing stress relaxation. A general viscoelastic response in rheology is defined for small deformations as

$$
\sigma_{i j}(t)=\int_{-\infty}^{t} \eta_{i j k l}(t-\tau) \frac{d u_{k l}}{d \tau} d \tau,
$$

where $\sigma_{i j}$ is the stress tensor and $u_{k l}$ corresponds to strains. $\eta_{i j k l}(t)$ is the generalization of the elasticity tensor to viscoelastic systems with a memory kernel. It is crucial to 
keep the tensor structure when symmetries are broken. Causality requires that $\eta_{i j k l}(t)$ is zero for negative $t$. $\eta_{i j k l}(t)$ captures the response to a constant shear rate applied to the material, in such a way that symmetries are preserved. We always imply a summation over repeated indices, which run over spatial $x$ and $y$ coordinates as we focus on twodimensional systems. Solids and liquids differ by the limiting value of the stress under a deformation, which, at long times, tends to zero for fluids and to a constant value for solids. As an equivalent description of viscoelastic response, one can consider the stress evolution as an input using the creep compliance tensor $c_{i j k l}(t)$ in the following way [40]:

$$
u_{i j}(t)=\int_{-\infty}^{t} c_{i j k l}(t-\tau) \frac{d \sigma_{k l}}{d \tau} d \tau .
$$

Creep is a progressive deformation of a material under constant stress. If it approaches a finite shear, the material is said to be solid, and if it increases linearly after a long time, the material is a fluid.

We are interested in linear viscoelasticity and focus on the simple case where the stress at the current time depends only on the current strain and strain rate. As a simple illustrative example, we note that, for the Kelvin-Voigt model of a solid, the relation between strain and stress is given by

$$
\sigma_{i j}=\kappa_{i j k l} u_{k l}+\eta_{i j m n} \dot{u}_{m n},
$$

where $\sigma_{i j}$ is the stress tensor, $u_{i j}$ is the strain tensor, $\dot{u}_{i j}$ is the strain rate tensor, $\kappa_{i j k l}$ is the elasticity tensor, and $\eta_{i j k l}$ is the viscosity tensor. Here $\sigma_{i j}$ and $u_{i j}$ are symmetric, and $\kappa_{i j k l}$ and $\eta_{i j m n}$ are symmetric in the first two and last two indices. Considering linear viscoelasticity and isotropy reduces the number of entries in $\kappa_{i j k l}$ and $\eta_{i j m n}$ to two in the parity-even case. These are the first and second Lamé parameters $\lambda$ and $\mu$ as well as two viscosities, bulk viscosity $\zeta$ and shear viscosity $\eta$. This approach was first proposed by Maxwell in his spring and damper model and later complemented by Kelvin, Voigt, and also Meyer [41] (see also [42-44] for modern developments).

In this Letter, following the same logic, we employ linearized viscoelasticity to construct parity-breaking generalized Kelvin-Voigt and Maxwell models in two dimensions that also break time-reversal symmetry, which we use to investigate the parity-odd viscoelastic response in solids and fluids. In the present case the parity breaking manifests itself through a nonzero value of the odd viscosity $\eta^{o}$ and the odd elastic coefficient $\kappa^{o}$. Odd elasticity encapsulates nonconservative microscopic interactions. This means that in a cyclic process the net elastic work $\oint \sigma_{i j} d u_{i j}$ can be nonzero in the presence of odd elasticity and that the sign of the net elastic work changes when the cyclic process is reversed. Therefore odd elasticity [18] breaks time-reversal symmetry and exists only in active systems $[17,18]$. Note that odd viscosity requires parity breaking but can exist in a passive system, as it is consistent with time reversibility [45].
Relaxation of parity-odd viscoelastic materials in two dimensions. - The relaxation times of a viscoelastic system tell us how long it takes for the material to return from a deformed state to its equilibrium state. In two dimensions there are two distinct types of stresses that one can apply to the material: shear and compression. As we shall see, parity breaking does not modify the compressional response. However, the response to shear receives contributions from odd transport coefficients. We are interested in determining the explicit form of the relaxation times. For the KelvinVoigt model we rewrite the constitutive equation in the following form:

$$
R_{i j k l} \dot{u}_{k l}=-u_{i j}+\kappa_{i j k l}^{-1} \sigma_{k l},
$$

where $R_{i j k l} \equiv \kappa_{i j m n}^{-1} \eta_{m n k l}$ and $i, j, k, l=\{1,2\}$, which we refer to as the relaxation-time tensor. In the Kelvin-Voigt model $u_{i j}$ corresponds to the deformation from a specific reference state. Analogously, for the Maxwell model in the presence of a flow given by the velocity $\vec{v}$ the constitutive relation reads

$$
R_{i j k l} \frac{d}{d t} \sigma_{k l}=-\sigma_{i j}+\eta_{i j k l} v_{k l},
$$

where $d / d t=\partial_{t}+v_{i} \partial_{i}$ is the convective derivative and $v_{k l}=\frac{1}{2}\left(\partial_{k} v_{l}+\partial_{l} v_{k}\right)$ is the symmetric part of the velocity gradients. In general the corotational part should also be included [13], but we omit it for simplicity. Four-index tensors possess a unique identity operator $\operatorname{Id}_{i j k l}=\delta_{i k} \delta_{j l}$, where $\delta_{i j}$ denotes the Kronecker symbol. In order to define $\kappa^{-1}$ we need to construct a tensor that, contracted with the elasticity tensor $\kappa_{m n k l}$, satisfies $\kappa_{i j m n}^{-1} \kappa_{m n k l}=\mathrm{Id}_{i j k l}$. We note that in classical elasticity the elasticity tensor is only partially invertible and we construct the inverse in the invertible subspace. Assuming that there is no memory in the system, we can use the symmetries to extract the structure of the relaxation-time tensor for an isotropic two-dimensional material

$$
\begin{aligned}
R_{i j k l}= & \tau_{1}\left(\delta_{i k} \delta_{j l}+\delta_{i l} \delta_{j k}\right)+\tau_{2} \delta_{k l} \delta_{i j} \\
& +\frac{\tau^{o}}{2}\left(\delta_{i k} \epsilon_{j l}+\delta_{i l} \epsilon_{j k}+\delta_{j k} \epsilon_{i l}+\delta_{j l} \epsilon_{i k}\right)
\end{aligned}
$$

in terms of the three coefficients $\left\{\tau_{1}, \tau_{2}, \tau^{o}\right\}$. Here $\epsilon_{i j}=-\epsilon_{j i}$ denotes the antisymmetric tensor with odd parity. On symmetry grounds the system exhibits three relaxation times. These times, $\tau_{1}=\left(\eta^{o} \kappa^{o}+\eta \mu\right) /\left\{4\left[\left(\kappa^{o}\right)^{2}+\mu^{2}\right]\right\}, \tau_{2}=(\zeta+\eta) /$ $[4(\lambda+\mu)]-\tau_{1}$, and $\left.\tau^{o}=\left(\eta^{o} \mu-\eta \kappa^{o}\right) /\left\{4\left[\left(\kappa^{o}\right)^{2}+\mu^{2}\right)\right]\right\}$, depend on the transport coefficients $\zeta, \lambda, \eta$, and $\mu$ defined below. $\tau_{1}$ and $\tau_{2}$ capture the relaxation of compression and shear, respectively. The relaxation time $\tau^{o}$ is associated with the relaxation of perpendicular responses depicted in Fig. 1 for the Kelvin-Voigt solid and in Fig. 2 for the Maxwell fluid. We note, however, that in general all of these relaxation 
(a)

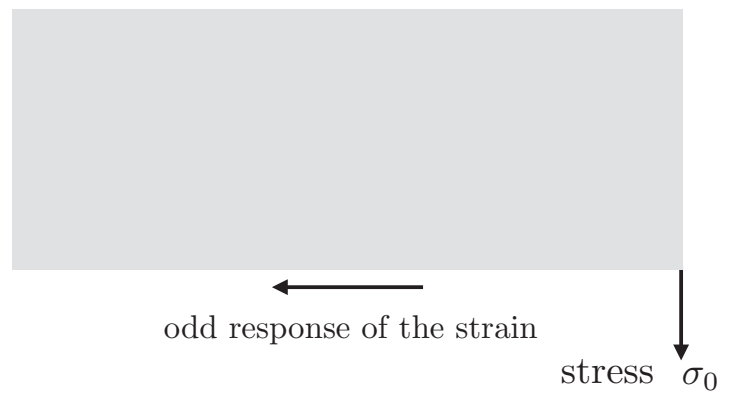

(b)

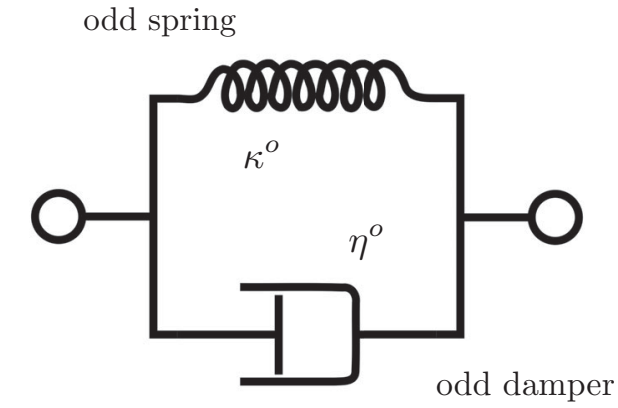

FIG. 1. (a) Sketch of the odd Kelvin-Voigt model. Upon application of a constant stress the resulting deformation will be perpendicular to the stress direction. The decay of the deformation will be captured by odd transport coefficients. (b) Depiction of the odd response in odd Kelvin-Voigt materials. It is modeled by a transverse spring and a damper connected in parallel.

processes are coupled, leading to an oscillatory relaxation dynamics.

In order to get a better physical understanding of the symmetry structure, it is convenient to use a basis of twodimensional matrices

$s^{0}=\left(\begin{array}{ll}1 & 0 \\ 0 & 1\end{array}\right), \quad s^{2}=\left(\begin{array}{cc}1 & 0 \\ 0 & -1\end{array}\right), \quad s^{3}=\left(\begin{array}{ll}0 & 1 \\ 1 & 0\end{array}\right)$.

In this basis the stress and displacements are defined as $\sigma^{\alpha}=s_{i j}^{\alpha} \sigma_{i j}, u^{\alpha}=s_{i j}^{\alpha} u_{i j}$, where $\alpha=\{0,2,3\}$; see Ref. [18] for details. The absence of $s^{1}=\left(\begin{array}{cc}0 & -1 \\ 1 & 0\end{array}\right)$ stems from the assumption that stress and deformation tensors are symmetric. In this representation the elastic tensor is given by a matrix with elements $\kappa^{\alpha \beta}=\frac{1}{2}\left(s^{\beta}\right)_{i j}^{-1} \kappa_{i j m n} s_{m n}^{\alpha}$. The original form can be obtained using the formula $\kappa_{i j m n}=$ $\frac{1}{2}\left(s^{\beta}\right)_{i j} \kappa^{\alpha \beta}\left(s^{\alpha}\right)_{m n}^{-1}$. In order to see the explicit form of the elastic tensors in these two bases, we start with the most general form of the elastic tensor,

$$
\begin{aligned}
\kappa_{i j k l}= & \mu\left(\delta_{i k} \delta_{j l}+\delta_{i l} \delta_{j k}\right)+\lambda \delta_{k l} \delta_{i j} \\
& +\frac{\kappa^{o}}{2}\left(\delta_{i k} \epsilon_{j l}+\delta_{i l} \epsilon_{j k}+\delta_{j k} \epsilon_{i l}+\delta_{j l} \epsilon_{i k}\right),
\end{aligned}
$$

and the viscosity tensor, (a)

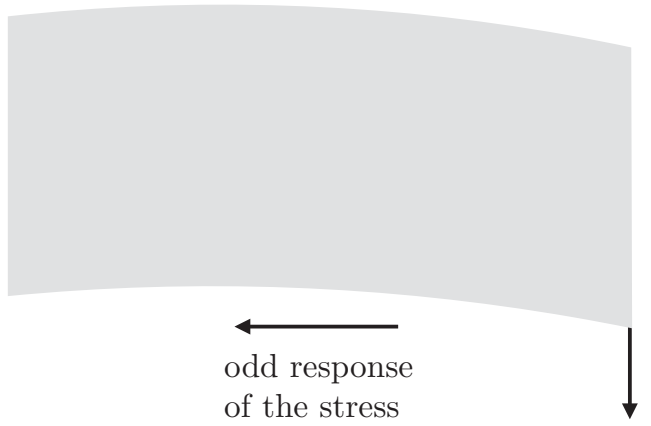

strain $u_{0}$

(b)

odd damper odd spring

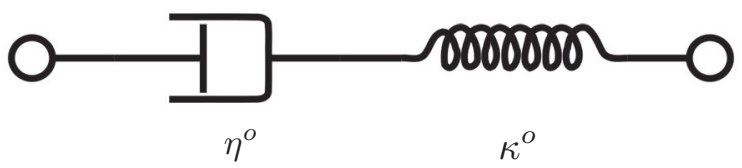

FIG. 2. (a) Sketch of the odd Maxwell model. A finite deformation will result in a transverse stress. Its relaxation will be captured by odd transport coefficients. (b) Depiction of the odd response in odd Maxwell materials. It is modeled by a spring and a damper connected in series. We emphasize that this collective representation does not imply that odd Maxwell fluids can be constructed out of springs and dampers.

$$
\begin{aligned}
\eta_{i j k l}= & \eta\left(\delta_{i k} \delta_{j l}+\delta_{i l} \delta_{j k}\right)+\zeta \delta_{k l} \delta_{i j} \\
& +\frac{\eta^{o}}{2}\left(\delta_{i k} \epsilon_{j l}+\delta_{i l} \epsilon_{j k}+\delta_{j k} \epsilon_{i l}+\delta_{j l} \epsilon_{i k}\right),
\end{aligned}
$$

which is consistent with spatial isotropy and the minor symmetry of indices. Using the representation in Eq. (7) we obtain

$$
\kappa^{\alpha \beta}=2\left(\begin{array}{ccc}
\lambda+\mu & 0 & 0 \\
0 & \mu & -\kappa^{o} \\
0 & \kappa^{o} & \mu
\end{array}\right)^{\alpha \beta}
$$

and

$$
\eta^{\alpha \beta}=2\left(\begin{array}{ccc}
\zeta+\eta & 0 & 0 \\
0 & \eta & -\eta^{o} \\
0 & \eta^{o} & \eta
\end{array}\right)^{\alpha \beta} .
$$

Now the inversion requires an inverse of a $3 \times 3$ matrix. The relaxation tensor in Eq. (6) has the simple form $R_{\alpha \beta}=\frac{1}{2} \kappa_{\alpha \gamma}^{-1} \eta_{\gamma \beta}$. It is also convenient to introduce an inverse of the relaxation-time tensor

$$
\Lambda_{i j k l}=\eta_{i j m n}^{-1} \kappa_{m n k l}
$$

that we call the relaxation rate tensor, whose matrix representation is $\Lambda_{\alpha \beta}=\frac{1}{2} \eta_{\alpha \gamma}^{-1} \kappa_{\gamma \alpha}=R_{\alpha \beta}^{-1}$. 
Modified Kelvin-Voigt material.-The first viscoelastic material we want to investigate is an active, chiral, viscoelastic solid (see Fig. 1). Such a material shall have parity-breaking viscous and elastic response transport coefficients together with the conventional parity preserving responses. It has been shown that odd elastic solids require some dissipation mechanism to make them stable [18]. Such a mechanism can be provided by a small viscosity. This type of viscoelastic solid was studied in Ref. [18]. In this Letter we want to focus on the interplay between odd elastic and odd viscous effects. To do that one can extend the Kelvin-Voigt model to account for paritybreaking responses. We start with the constitutive equation (3) written in the matrix representation:

$$
\sigma_{\alpha}=\kappa_{\alpha \beta} u_{\beta}+\eta_{\alpha \gamma} \dot{u}_{\gamma} .
$$

In this model the stresses due to viscosity and elasticity are additive and they can be represented by a spring and a damper in parallel. Odd elastic solids have been modeled by using point masses connected by springs that have linear but noncentral forces [18,46,47], the odd Kelvin-Voigt model is the simplest extension of such solids to include damping or viscous effects.

The resulting odd response in the continuum limit is perpendicular to the applied force. In the case of viscoelastic solids modeled by the Kelvin-Voigt equation (13) we are interested in the relaxation of displacement. To understand this we have to analyze the eigenvalues of the relaxation-time tensor. We get one time that corresponds to compression that is real and does not depend on paritybreaking coefficients,

$$
\tilde{\tau}=\frac{1}{2} \frac{\eta+\zeta}{\mu+\lambda},
$$

and two complex times that determine the relaxation of the deviatoric perturbations,

$$
\tilde{\tau}_{1,2}=\frac{1}{2} \frac{\eta^{o} \kappa^{o}+\eta \mu \pm i\left(\eta^{o} \mu-\eta \kappa^{o}\right)}{\mu^{2}+\left(\kappa^{o}\right)^{2}} .
$$

We conclude that in the parity-breaking viscoelastic solids shear perturbations create damped oscillating waves noticed in Ref. [18] without odd or Hall viscosity. We also note that in the limit with $\kappa^{o}=0$ the waves still exist, and the imaginary part is given by the product of odd viscosity and an even elastic coefficient $\mu$.

Modified Maxwell material.-Soft materials like dilute biopolymer solutions can also exhibit viscoelastic behavior [48-51]. They can transiently store elastic energy, but they can also flow like viscous fluids.

We would like to have a model that can serve as a description of chiral active polymer solutions with odd viscoelastic terms. We follow a phenomenological path by taking a Maxwell model, in which $\sigma_{i j}=\sigma_{i j}^{D}=\sigma_{i j}^{S}$, $u_{k l}=u_{k l}^{D}+u_{k l}^{S}$, and supplement it with parity-breaking terms. The superscripts $D$ and $S$ refer to a damper and a spring, respectively. In the Maxwell model the strain is additive and modeled by a spring and a damper in series (as opposed to being parallel in the Kelvin-Voigt model), as presented in Fig. 2. The constitutive equation together with the momentum conservation equation for the Maxwell fluid reads (see also Fig. 2)

$$
\begin{gathered}
v_{k l}=\eta_{i j k l}^{-1} \sigma_{i j}+\kappa_{i j k l}^{-1} \frac{d}{d t} \sigma_{i j}, \\
\rho \frac{d}{d t} v_{i}=\partial_{j} \sigma_{i j},
\end{gathered}
$$

where $\rho$ is the mass density. We can rewrite the above equations using the inverse of the relaxation-time tensor (12). This model together with the relaxation-time analysis for broken parity and time-reversal symmetries is a central result of our Letter. We perform analytic studies in a simplifying limit. We consider an analytically tractable limit, taking small elastic coefficients $\kappa_{i j k l}$ while keeping the relaxation rates $\Lambda_{i j m n}$ fixed. The relaxation times are given again by the expressions (14) and (15). In a complete analogy with Kelvin-Voigt material we see that changing the volume of the material relaxes without any influence from activity. On the other hand, area preserving shear deformations lead to oscillating, damped shear waves.

Compressible Maxwell fluids.-Parity breaking affects pressure in a significant way [33]. We can investigate this in the compressible regime of the odd Maxwell model. Let us now look at Eq. (16a) linearized around a solution with no velocity and $\rho=\rho_{0}$ governing the dynamics of an odd viscoelastic Maxwell fluid:

$$
\begin{aligned}
\frac{\partial}{\partial t} \sigma_{i j}= & \mu\left(\partial_{i} v_{j}+\partial_{j} v_{i}\right)+\lambda \delta_{i j} \partial_{k} v_{k} \\
& +\frac{\kappa^{o}}{4}\left(\partial_{i}^{*} v_{j}+\partial_{i} v_{j}^{*}+\partial_{j}^{*} v_{i}+\partial_{j} v_{i}^{*}\right)-\Lambda_{i j m n} \sigma_{m n}, \\
\rho_{0} \frac{\partial}{\partial t} v_{i}= & \partial_{j} \sigma_{i j}, \\
\frac{\partial}{\partial t} \delta \rho= & -\rho_{0} \partial_{k} v_{k},
\end{aligned}
$$

where $\partial_{i}^{*}=\epsilon_{i j} \partial_{j}$ and $v_{i}^{*}=\epsilon_{i j} v_{j}, \rho=\rho_{0}+\delta \rho$, and $p=$ $-\frac{1}{2} \sigma_{i}^{i}$ (see also Ref. [52]). In order to make analytic progress we take a limit $\eta^{o} / \eta \rightarrow 0$ and $\zeta / \eta \rightarrow 0$ simultaneously. Next we rewrite the resulting equations using vorticity $\Omega=$ $\epsilon_{i j} \partial_{i} v_{j}$ and the divergence $\Theta=\partial_{k} v_{k}$ as variables:

$$
\begin{gathered}
\rho_{0} \frac{\partial^{2}}{\partial t^{2}} \Omega=\mu \nabla^{2} \Omega-\kappa^{o} \nabla^{2} \Theta, \\
\rho_{0} \frac{\partial^{2}}{\partial t^{2}} \Theta=(2 \mu+\lambda) \nabla^{2} \Theta+\kappa^{o} \nabla^{2} \Omega .
\end{gathered}
$$



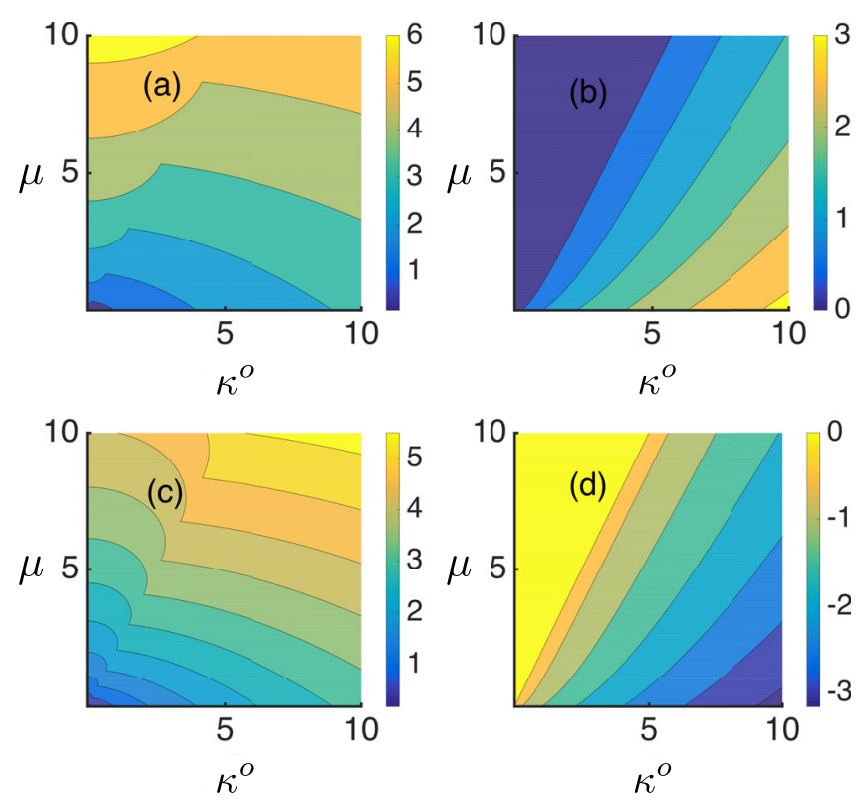

FIG. 3. Real and imaginary parts of the frequency eigenvalues. (a),(b) Real and imaginary parts, respectively, of the first eigenvalue. (c),(d) Real and imaginary parts, respectively, of the second eigenvalue. These are obtained for the parameters $k=1, \lambda=0$, and $\rho_{0}=1 / 2$.

These equations allow vorticity and divergence sound waves. Now we study the plain-wave perturbations $\Omega(t, \vec{x})=$ $\Omega(\omega, \vec{k}) e^{i(\omega t+\vec{k} \cdot \vec{x})}$ and $\Theta(t, \vec{x})=\Theta(\omega, \vec{k}) e^{i(\omega t+\vec{k} \cdot \vec{x})}$. In the usual viscoelastic theory, longitudinal waves corresponding to the compressional disturbances are decoupled from the transverse waves that correspond to shear perturbations [53]. Odd viscoelasticity couples these two collective disturbances. These linear waves have dispersion relations given by

$\omega= \pm k \frac{1}{\sqrt{2 \rho_{0}}}\left[(\lambda+3 \mu) \pm\left((\lambda+\mu)^{2}-4 \kappa^{o 2}\right)^{1 / 2}\right]^{1 / 2}$.

We obtain two modes for each equation. The eigenvalues are plotted in Fig. 3. Now, considering the extreme case of $\kappa^{o} \gg \lambda, \mu$, we have

$$
\omega= \pm k \frac{1}{\sqrt{\rho_{0}}} \sqrt{\kappa^{o}}( \pm i)^{1 / 2}
$$

The above dispersion relation yields waves that have speeds proportional to $\sqrt{\kappa^{\circ}}$ and also linear damping proportional to $\sqrt{\kappa^{\circ}}$ in the stable case with $\kappa^{o}>0$. They are distinct from Avron waves in fluids with odd viscosity [31], which are of diffusive nature. As such they provide a novel manifestation of parity-breaking excitations in hydrodynamics.

Discussion.-We have focused our study of odd viscoelastic responses, in chiral active matter, on two-dimensional systems. Our motivation comes from a particular pertinence of chiral asymmetries near surfaces as in two dimensions. An important example of such an active chiral system in biology is the cell cortex. This is a thin layer of an active gel formed by actin filaments and many other protein components which is located below the cell membrane. This gel layer has viscoelastic properties because filaments turn over in about one minute, giving its elastic properties on short timescales and viscous behaviors at long times [49]. It has been shown that this system generates active stresses in the layer and active chiral processes give rise to asymmetric flows [54-56]. Other examples of two-dimensional viscoelastic systems include interfaces between complex active fluids, thin layers of viscoelastic substances placed between plates, and solid metamaterials constructed with active or nonreciprocal microscopic interactions, as described in Ref. [47], where the Kelvin-Voigt model of viscoelasticity is directly applicable.

Odd active viscoelasticity can, in principle, be tested in microrheological experiments, where viscoelastic properties of complex fluids can be determined from the motion of embedded colloidal particles [57]. Based on symmetry arguments, the dynamics of a tracer particle in an odd viscoelastic medium would be described by an equation of the form

$m \dot{V}_{i}(t)=F_{i}-\int_{0}^{t} d t^{\prime}\left(\begin{array}{cc}\eta\left(t-t^{\prime}\right) & -\eta^{o}\left(t-t^{\prime}\right) \\ \eta^{o}\left(t-t^{\prime}\right) & \eta\left(t-t^{\prime}\right)\end{array}\right)_{i j} V_{j}\left(t^{\prime}\right)$

Here $\vec{V}$ is the velocity of the bead of mass $m, \vec{F}$ denotes an applied force, and the memory kernels $\eta(t)$ and $\eta^{o}(t)$ capture normal and odd viscoelastic responses, respectively. In a parity-breaking viscoelastic fluid the drift of a particle should be governed by odd transport coefficients. This suggests that signatures of effects associated with odd viscoelasticity could be observed in the dynamics of particles embedded in chiral active gels.

P. S. was supported by the DFG through the Leibniz Program, the cluster of excellence ct.qmat (EXC 2147, Project No. 390858490), and the National Science Centre (NCN) Sonata Bis Grant No. 2019/34/E/ST3/00405. V. V. was supported by the Complex Dynamics and Systems Program of the Army Research Office under Grant No. W911NF-19-1-0268 and the Simons Foundation. This work was partially supported by the University of Chicago Materials Research Science and Engineering Center, which is funded by National Science Foundation under Grant No. DMR-2011854.

*surowka@pks.mpg.de

[1] R. Lakes, Viscoelastic Materials (Cambridge University Press, Cambridge, England, 2010).

[2] I. M. Ward, An Introduction to the Mechanical Properties of Solid Polymers (Wiley, New York, 2004).

[3] K. Bertoldi, V. Vitelli, J. Christensen, and M. van Hecke, Nat. Rev. Mater. 2, 17066 (2017). 
[4] S. Shankar, A. Souslov, M. J. Bowick, M. C. Marchetti, and V. Vitelli, arXiv:2010.00364.

[5] K. Kruse, J. F. Joanny, F. Jülicher, J. Prost, and K. Sekimoto, Phys. Rev. Lett. 92, 078101 (2004).

[6] S. Fürthauer, M. Strempel, S. W. Grill, and F. Jülicher, Eur. Phys. J. E 35, 89 (2012).

[7] J.-C. Tsai, F. Ye, J. Rodriguez, J. P. Gollub, and T. C. Lubensky, Phys. Rev. Lett. 94, 214301 (2005).

[8] C. L. Henley, AIP Conf. Proc. 1134, 54 (2009).

[9] L. N. Vandenberg and M. Levin, Semin. Cell Dev. Biol. 20, 456 (2009).

[10] C. L. Henley, J. Stat. Phys. 148, 741 (2012).

[11] S. Fürthauer, M. Strempel, S. W. Grill, and F. Jülicher, Phys. Rev. Lett. 110, 048103 (2013).

[12] S. R. Naganathan, S. Fürthauer, M. Nishikawa, F. Jülicher, and S. W. Grill, eLife 3, e04165 (2014).

[13] F. Jülicher, S. W. Grill, and G. Salbreux, Rep. Prog. Phys. 81, 076601 (2018).

[14] M. C. Marchetti, J. F. Joanny, S. Ramaswamy, T. B. Liverpool, J. Prost, M. Rao, and R. A. Simha, Rev. Mod. Phys. 85, 1143 (2013).

[15] S. Ramaswamy, Annu. Rev. Condens. Matter Phys. 1, 323 (2010).

[16] D. Banerjee, A. Souslov, A. G. Abanov, and V. Vitelli, Nat. Commun. 8, 1573 (2017).

[17] G. Salbreux and F. Jülicher, Phys. Rev. E 96, 032404 (2017).

[18] C. Scheibner, A. Souslov, D. Banerjee, P. Surówka, W. T. M. Irvine, and V. Vitelli, Nat. Phys. 16, 475 (2020).

[19] A. Mitra and M. Lenz, Nat. Commun. 10, 920 (2019).

[20] A. Maitra and S. Ramaswamy, Phys. Rev. Lett. 123, 238001 (2019).

[21] L. A. Hoffmann, K. Schakenraad, R. M. H. Merks, and L. Giomi, Soft Matter 16, 764 (2020).

[22] Y. Sumino, K. H. Nagai, Y. Shitaka, D. Tanaka, K. Yoshikawa, H. Chaté, and K. Oiwa, Nature (London) 483, 448 (2012).

[23] Y. Tabe and H. Yokoyama, Nat. Mater. 2, 806 (2003).

[24] A. P. Petroff, X.-L. Wu, and A. Libchaber, Phys. Rev. Lett. 114, 158102 (2015).

[25] I. H. Riedel, K. Kruse, and J. Howard, Science 309, 300 (2005).

[26] J. Denk, L. Huber, E. Reithmann, and E. Frey, Phys. Rev. Lett. 116, 178301 (2016).

[27] P. Lenz, J.-F. Joanny, F. Jülicher, and J. Prost, Phys. Rev. Lett. 91, 108104 (2003).

[28] N. Uchida and R. Golestanian, Phys. Rev. Lett. 104, 178103 (2010).

[29] B. C. van Zuiden, J. Paulose, W. T. M. Irvine, D. Bartolo, and V. Vitelli, Proc. Natl. Acad. Sci. U.S.A. 113, 12919 (2016).
[30] J. E. Avron, R. Seiler, and P. G. Zograf, Phys. Rev. Lett. 75, 697 (1995).

[31] J. E. Avron, J. Stat. Phys. 92, 543 (1998).

[32] M. F. Lapa and T. L. Hughes, Phys. Rev. E 89, 043019 (2014).

[33] A. Lucas and P. Surówka, Phys. Rev. E 90, 063005 (2014).

[34] E. Kogan, Phys. Rev. E 94, 043111 (2016).

[35] S. Ganeshan and A. G. Abanov, Phys. Rev. Fluids 2, 094101 (2017).

[36] Z. Liao, M. Han, M. Fruchart, V. Vitelli, and S. Vaikuntanathan, J. Chem. Phys. 151, 194108 (2019).

[37] A. Souslov, A. Gromov, and V. Vitelli, Phys. Rev. E 101, 052606 (2020).

[38] A. Souslov, K. Dasbiswas, M. Fruchart, S. Vaikuntanathan, and V. Vitelli, Phys. Rev. Lett. 122, 128001 (2019).

[39] V. Soni, E. S. Bililign, S. Magkiriadou, S. Sacanna, D. Bartolo, M. J. Shelley, and W. T. M. Irvine, Nat. Phys. 15, 1188 (2019).

[40] A. C. Pipkin, Lectures on Viscoelasticity Theory (Springer, New York, 1986).

[41] N. Phan-Thien, Understanding Viscoelasticity: An Introduction to Rheology (Springer, New York, 2012).

[42] T. Azeyanagi, M. Fukuma, H. Kawai, and K. Yoshida, Phys. Lett. B 681, 290 (2009).

[43] M. Fukuma and Y. Sakatani, Phys. Rev. E 84, 026316 (2011).

[44] J. Armas and A. Jain, J. High Energy Phys. 01 (2020) 126.

[45] C. Hoyos, Int. J. Mod. Phys. B 28, 1430007 (2014).

[46] D. Zhou and J. Zhang, Phys. Rev. Research 2, 023173 (2020).

[47] C. Scheibner, W. T. M. Irvine, and V. Vitelli, Phys. Rev. Lett. 125, 118001 (2020).

[48] J. Prost, F. Jülicher, and J.-F. Joanny, Nat. Phys. 11, 111 (2015).

[49] E. Fischer-Friedrich, Y. Toyoda, C. J. Cattin, D. J. Müller, A. A. Hyman, and F. Jülicher, Biophys. J. 111, 589 (2016).

[50] A. F. Pegoraro, P. Janmey, and D. A. Weitz, Cold Spring Harbor Perspect. Biol. 9, a022038 (2017).

[51] D. Saintillan, Annu. Rev. Fluid Mech. 50, 563 (2018).

[52] P. C. Bollada and T. N. Phillips, Arch. Ration. Mech. Anal. 205, 1 (2012).

[53] R. D. Borcherdt, Viscoelastic Waves in Layered Media (Cambridge University Press, Cambridge, England, 2009).

[54] G. Salbreux, J. Prost, and J.-F. Joanny, Phys. Rev. Lett. 103, 058102 (2009).

[55] J. S. Bois, F. Jülicher, and S. W. Grill, Phys. Rev. Lett. 106, 028103 (2011).

[56] P. Gross, K. V. Kumar, N. W. Goehring, J. S. Bois, C. Hoege, F. Jülicher, and S. W. Grill, Nat. Phys. 15, 293 (2019).

[57] T. M. Squires and T. G. Mason, Annu. Rev. Fluid Mech. 42, 413 (2010). 